(c) 2010 IEEE. Personal use of this material is permitted. Permission from IEEE must be obtained for all other uses, in any current or future media, including reprinting/republishing this material for advertising or promotional purposes, creating new collective works, for resale or redistribution to servers or lists, or reuse of any copyrighted component of this work in other works. 


\title{
Semantic Service Retrieval and QoS Measurement in the Digital Ecosystem Environment
}

\author{
Hai Dong, Farookh Khadeer Hussain, Elizabeth Chang \\ Digital Ecosystems and Business Intelligence Institute \\ Curtin University of Technology \\ Perth, Australia \\ e-mail: \{hai.dong, farookh.hussain, elizabeth.chang\}@cbs.curtin.edu.au
}

\begin{abstract}
Digital Ecosystem is an innovative high-tech environment with the purpose of supporting the activities among species within the business ecosystem. In this paper, we concern about the research issue of service retrieval within such an environment. Due to the fact that species are heterogeneous and geographically dispersed, to precisely and quickly locate a service provider becomes an issue. In addition, the Digital Ecosystem environment urgently requires the structualization of service information and a set of unified QoS measurement for service ranking and evaluation. In order to unfold the issues in detail, we use the means of case study and literature survey. Eventually we formulate the research issues in this domain and provide a possible solution.
\end{abstract}

Keywords-component; Digital Ecosystems, measurement metrics, quality of services (QoS), semantic web technologies, service retrieval

\section{INTRODUCTION}

Digital Ecosystem is a novel concept emerging with the appearance of business ecosystem. The goal of Digital Ecosystem is to improve the efficiency of the communications between internal agents and to structuralize the business ecosystem [1]. In this paper, we will focus on the issue of semantic service retrieval and QoS measurement in the Digital Ecosystem environment. By means of a case study and literature survey, we will explore and formulate the research issues in this domain. Furthermore, we will present a state-of-the-art solution against these discovered issues.

This paper is the extended version of our IT Revolutions 2008 conference paper [2]. While our conference paper deals with Service Oriented Environment (SOE), the current paper deals with the Digital Ecosystem environment, which belongs to a different conceptual category. New materials include the introduction of Digital Ecosystem (Section 2), new case study for studying the motivation of this research (Section 3), newly defined research issues (Section 4) and new survey of existing literature (Section 5) and a proposed solution (Section 6).

The rest of this paper is organized as follows: first of all we will introduce the origination and conceptual framework of the Digital Ecosystem and present the issues within the field; following that we attempt to explore the research motivations in this field by a case study; then by means of a thorough survey on the existing literature in the field of semantic service retrieval and QoS measurement, we will discover the issues in the current research field; next we will formulate the research issues in the Digital Ecosystem environment; later on against the issues we will propose and present a solution in the air; finally we will draw the conclusions and plan our future works.

\section{DigitAL ECOSYSTEM}

It is widely recognized that individuals and organizations within the business world is like species in the ecosystem, in which species have to compete and cooperate with others for survival, and thus the business world is named as Business Ecosystem by Moore [3], which is defined as "an economic community supported by a foundation for interacting organizations and individuals". However, the existing technologies within the Business Ecosystem are not capable of supporting the interactivities among species. As a result, a proposed new technical environment - Digital Ecosystem emerges.

Digital Ecosystem is defined as "an open, loosely coupled, domain clustered, demand-driven, self-organizing and agent-based environment, in which each species is proactive and responsive for its own benefit and profit" [4]. From its definition, it can be observed that Digital Ecosystem primarily comprises of two parts - species and Digital Ecosystem environment. Species are the inhabitants of the Digital Ecosystem, which can be basically divided into three categories - biological species, e.g. human, economic species, e.g. companies and organizations, and digital species, e.g. agents. These species need to interact with others in order to gain benefits and profits, e.g., a company needs to implement a series of business transactions with customers who could be individuals or other companies in order to gain profits. On the other hand, the Digital Ecosystem environment facilitates the interactivities through underlying infrastructures, technologies and services in order to support these species' survival.

Subsequently let us concern about the interactivities within the Digital Ecosystem. These interactivities involve goods and service transactions, and data transmission, etc [4, 5]. In particular, in the interactivities of service transactions, species can play dual roles - service requester and service provider. However, three issues emerge here as follows: 
1) Since species are heterogeneous and geographically dispersed in an ecosystem [4], how to locate a service provider by a given service is a critical function of the Digital Ecosystem.

2) The service information in an ecosystem is heterogeneous and a lack of semantic structure. Hence, how to standardize and structuralize the service information becomes the crucial requirement of the Digital Ecosystem.

3) There is a lack of a set of unified metrics to measure QoS. Therefore, how to evaluate QoS is another functional requirement of the Digital Ecosystem

In the next section, we will reveal the motivations for the three research issues above in the field of Digital Ecosystem, by means of a case study.

\section{CASE StUdy FOR STUdying THE Motivations OF SEMANTIC SERVICE RETRIEVAL AND QOS MEASUREMENT \\ RESEARCH IN THE DIGITAL ECOSYSTEM ENVIRONMENT}

In this section, by means of a case study, we will analyse the research motivations in the fields of semantic service retrieval and QoS measurement.

John is a farmer and lives in Perth (capital city of Western Australia) and desires a sheep removal service provided by a local company, in order to help him to move sheep from Perth to City B. In addition, John intends to find out the ranking of all available sheep removal companies in Perth based on QoS.

From the perspective of the internet services, there are two primary categories of service search engines that can be found by John.

The first category is generic search engines, such as Yahoo! ${ }^{\circledR}$ and Google ${ }^{\mathrm{TM}}$. For example, John can enter "sheep removal companies in Perth" into a generic search engine (here the example is Yahoo!®). From the retrieved results from the search engine (Fig. 1), it is observed that most of the retrieved results do not match John's search intention sheep removal companies in Perth, and the service information is difficult to be distinguished and identified from the results. Thus, it is asserted that the performance of the generic search engine is poor in this case study.

The reasons behind the poor performance of the search engine can be concluded as follows:

- The search engine uses traditional keywords-based search strategy without incorporating or taking into account semantic web technologies to assist the search engine to fully understand the sense of the user's query words. This causes the poor performance of the search engine in precision.

- The generic search engine is not specially designed for the purpose of service retrieval. As a result of this, the search process has to be carried out against a much larger information source. Due to this reason and due to the fact the search process is keywordbased the retrieved search results are not accurate and do not consider the context of the search query.

- The format of the retrieved service information is not standardized, which makes users difficult to read and comprehend the retrieved service information.

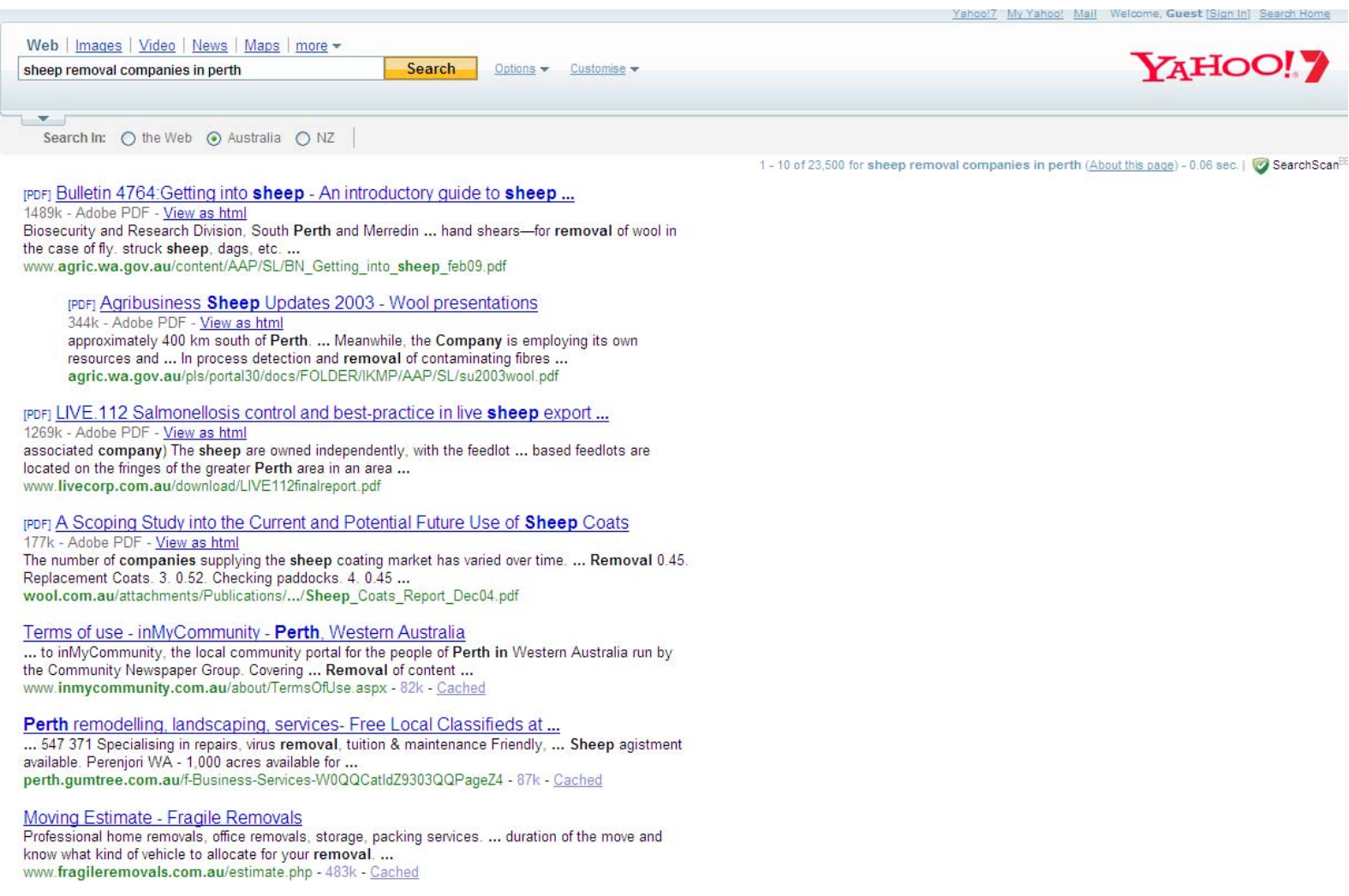

Figure 1. Retrieved results of "sheep removal companies in Perth" from Yahoo search engine 


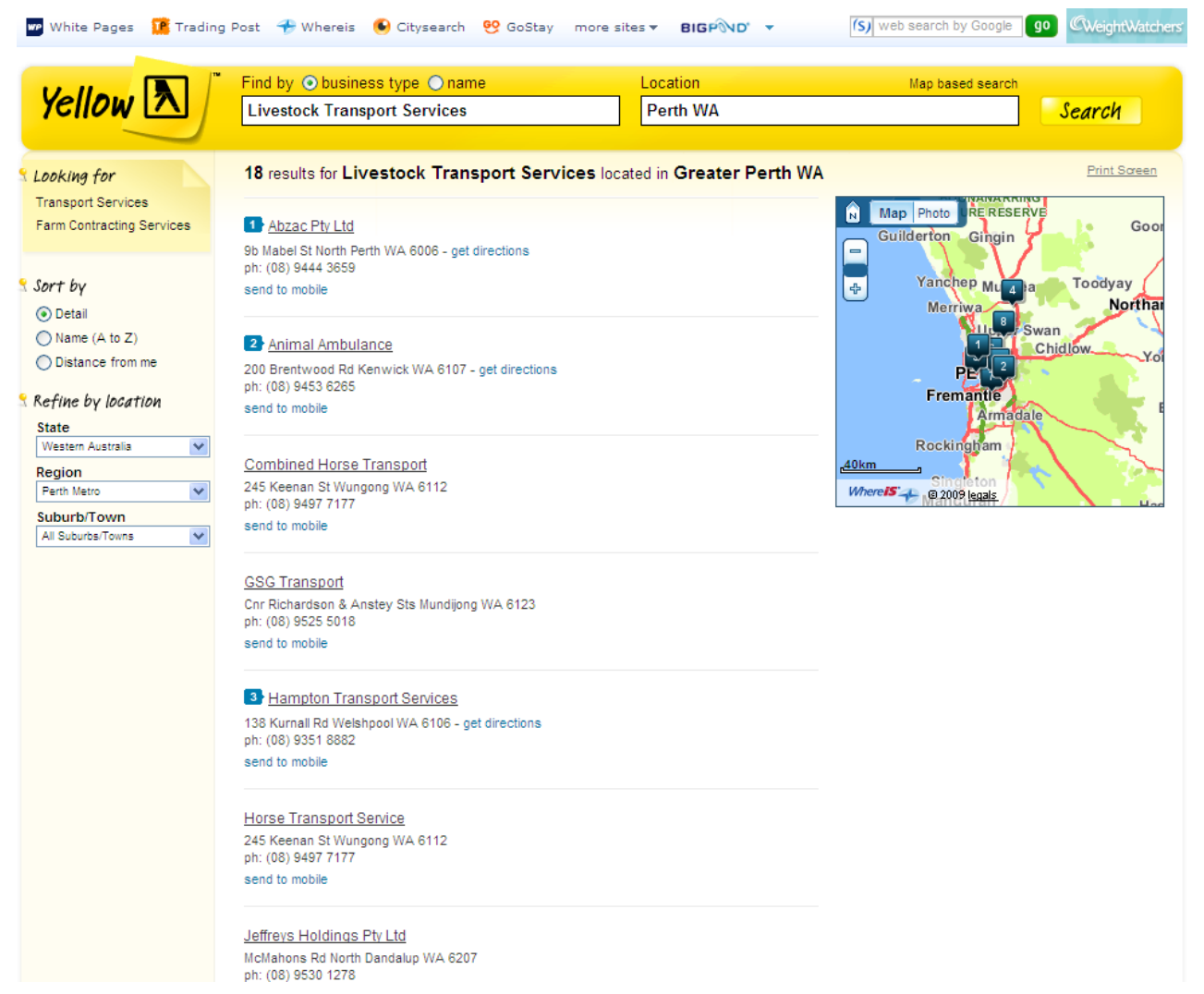

Figure 2. Businesses under the category of "livestock transport service in Perth" in Australian Yellowpages website

An enhanced approach is that John can access into a repository of local business directory, such as Yahoo! ${ }^{\circledR}$ or Google $^{\mathrm{TM}}$ local search, online Yellowpages. These local search engines (here the example is Australian online Yellowpages ${ }^{\circledR}$ ) normally can provide John with two options of service retrieval as follows:

- One option is that John can browse businesses under the "livestock transport services" category in the location "Perth WA", by following the "browse by category" (Fig. 2). This style can provide John with more precise search results and structured service information. The defect is that John needs to follow the whole category of the website step by step, which is expensive in terms of time and effort.

- Another option is that John can directly enter "sheep removal" into the business type box and "Perth" into the location box of the search engine provided by the website (Fig. 3). This can save its searching time, but this approach has its own disadvantages as well - the search engine cannot understand the user's query intention and thus returns non-relevant results. Similar to the generic search engines, the reason behind this is that the local search engine does not use semantic web technologies to help users to denote their searching concepts.

Apart from the lack of semantic web technologies' support, another limitation of the local service search engines is that John cannot find out which company has better performance from the perspective of sheep removal service.
The reason behind that is that these search engines do not provide user-oriented QoS measurements.

Based on the above case study, it is observed that both of the generic and local search engines are far from perfect, when searching for a given service. The research motivations in the field of semantic service retrieval and QoS measurement in the Digital Ecosystem environment can be concluded as follows:

- Designing multi-domains or domain-specific service search engines with semantic web technologies enhancing the search precision (and);

- Designing user-oriented QoS Measurement metrics for evaluating and ranking retrieved service information.

In the next section, following the two research motivations, we will make a brief survey on the status of current research in this field.

\section{RELATED WORKS}

In the section, we briefly review the current literature with respect to semantic service retrieval and QoS measurement and analyze their limitations.

\section{A. Semantic Service Retrieval}

While there are a great number of semantic search engines being developed (e.g., SWoogle, TAP), few of them attempt to provide optimized solutions for the service retrieval field. 


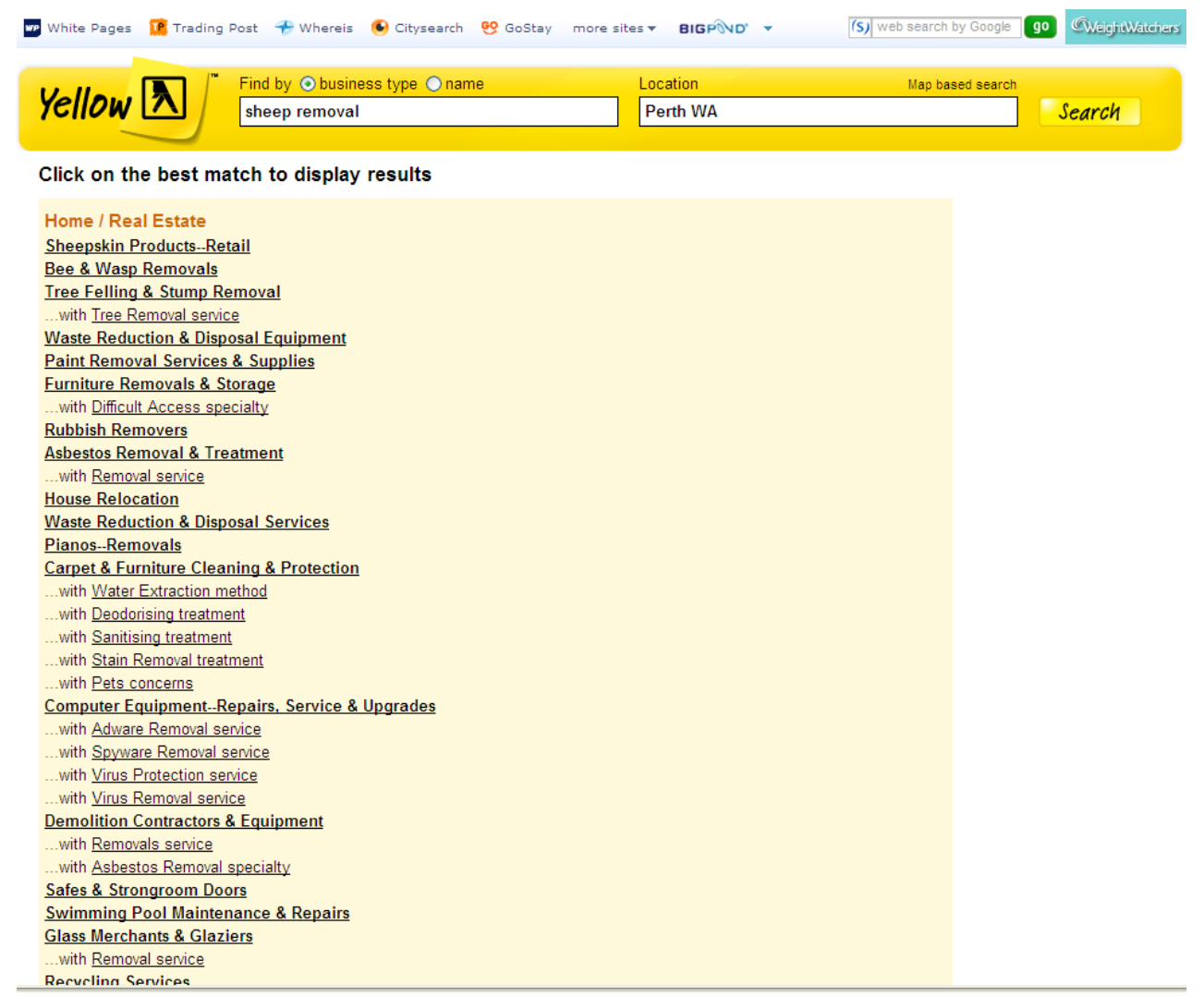

Figure 3. Retrieved results from online Australian Yellowpages search engine based on query words "sheep removal"

Liu et al. [6] developed an e-service platform integrated with semantic search for e-service metadata. E-service metadata refers to the descriptions of e-services and providers, which is adopted to publish and to discover eservices. There are two types of metadata in the system: business level metadata - the description of e-service providers, and service level metadata - the description of basic information about e-service. The authors adopt Universal Description, Discovery and Integration (UDDI) which is a web service standard to register and search eservices. Three means for searching service and business are provided, which are find_business, find_service and XQuery. Find_business is to return a list of service providers for specific conditions; find service is to return the information for a list of services who match customized conditions; XQuery is to query extended metadata added in a businessService list.

The limitations of the e-service search engine can be concluded as follows:

- Only one-tier (service categories-services) concept hierarchy cannot reflect the complex relationships between services in the Digital Ecosystem environment (and);

- There is no methodology provided for the concept hierarchy update in order to adapt for the change in service environment (and);

- The volume of its knowledge-base seems so limited that it only can be applied in limited fields (and);
- There is no QoS measurements provided for the querying results, which could lead to unorganized data structure and presentation to the user.

\section{B. QoS Measurement}

QoS is defined by ITUT Study Group as "a set of quality requirements on the collective behavior of one or more objects" [7]. In the field of computer networking and telecommunication, QoS concerns bout the ability of providing different priority to different applications or user, or guaranteeing a certain level of performance. While QoS development has achieved considerable progress in the field of distributed platforms, operation systems, transport systems and multimedia networking [8], few researches focus on service retrieval for the business ecosystem and integration of service retrieval and QoS measurement. In the rest of this section, we will introduce some typical QoS measurements in the service domain.

Gekas [9] propose a set of metrics for web service ranking. Four main categories of ranking strategies are provided by these metrics, which are degree-based rankings that calculate the percentage of fed services in each web service, hubs-authorities-based rankings that calculate the ratio between the number of incoming services and the number of outgoing services, non-functional rankings that focus on the NFPs of web service, and non-connectivity rankings that focus on the connectivity of web service networks. Similarly, Menascé [10] defines a set of metrics 
for web service quality management, including availability that is the time proportion of a service operation, security that concerns information confidentiality, response time and throughput that is the percentage that a service can process requests.

Roman et al. [11] design a Web Service Modeling Ontology (WSMO) approach that recommends a set of nonfunctional properties for each particular element of a web service description. Toma et al. [9] propose a web service evaluation system based on two different evaluation strategies. One strategy is to use the WSMO to describe the values of NFPs of web service, such as QoS, Service Level Agreement (SLA) etc. Hence, web services can be ranked according to the values of user-preferred NFP. Another strategy is a multi-criteria evaluation, which considers evaluating multiple NFPs from three main perspectives - the user-preferred NFPs, the level of importance of the NFPs, and the ascending or descending order of services.

A number of researches focus on the web service discovery with QoS measurements. Zhang et al. [12] concern about measuring QoS for service discovery in the universal network. Universal network combines Telecom network with IP network in order to provide services to clients. A series of metrics are developed for QoS measurement for services provided in the universal network, including connectivity, security, trustworthy degree, delay, loss probability, and QoS spectrum. The QoS metrics are defined by OWL with the purpose of sharing the QoS knowledge when discovering services in the universal network. Similarly, Pan [13] concerns using QoS as constraints for discovering web services. Four aspects of QoS metrics are considered, which are runtime-related QoS metrics, e.g., scalability, capacity, transaction support-related QoS metrics, e.g., integrity, atomicity, configuration management and cost-related QoS metrics, e.g., regulatory, cost, and security-related QoS metrics, e.g., authentication, authorization.

The limitations of the above QoS researches can be concluded as follows:

- None of the researches concerns about the services in the Digital Ecosystem environment, as they all focus on web services. The services in the Digital Ecosystems contain diverse forms, e.g., food services, business services, web services, etc., which is broader than it in the web service environment.

- None of the researches integrate service retrieval with QoS measurements.

- Few of the researches concern user-oriented QoS measurement, e.g. trustworthiness, confidence etc. As users are the eventual receivers of the services, their perception towards QoS should have reasonable impact on the measurement.

As can be clearly seen from the discussion above, research is being carried out independently in the fields of both of the semantic service retrieval and QoS measurement, without any attempt to integrate them together. In the next section, we define this issue formally.

\section{RESEARCH ISSUES FORMULATION}

In this paper, we combine our findings from the previous case study (Section 3) and our review of the existing literature (Section 4) and define the research issues in the field of semantic service retrieval and QoS measurement. The core research question is:

How to design a multi-domain or specific domain service search engine in the Digital Ecosystem environment enhanced by semantic web technologies and QoS measurements?

The potential research issues under this core research question are:

- For multi-domain or specific domain service retrieval, multi-domain or specific domain service ontologies need to be designed according to the particular service domain knowledge in the Digital Ecosystem environment.

- Multi-domain or specific domain service metadata format needs to be designed according to the service domain knowledge in the Digital Ecosystem environment, in order to standardize service metadata.

- Conceptual frameworks of multi-agents needs to be designed to harvest service metadata from the Digital Ecosystem environment according to the multi-domain or specific domain service ontologies and metadata formats.

- Multi-agent communication mechanisms need to be designed to improve harvest efficiency.

- Mechanisms needs to be designed for updating the knowledge-bases that store the multi-domain or specific domain service ontologies, in order to allow the ontologies to adapt to the dynamic change of knowledge in all service domains of the Digital Ecosystem.

- Search algorithms need to be designed based on the multi-domain and specific domain service ontologies, in order to realize the enhancement from multiple perspectives of search engine performance.

- A set of unified metrics needs to be designed to measure QoS for the diverse services in the Digital Ecosystem environment.

- Domain-specific service quality measurement criteria need to be designed according to the specific service domain knowledge in order to cooperate with the unified metrics to measure QoS in the Digital Ecosystem environment.

- Mechanisms need to be designed for updating the domain-specific service quality measurement criteria, in order to allow the criteria to adapt to the dynamic change of knowledge in the service domains of Digital Ecosystem.

- A conceptual framework for integrating the semantic service search engine and the QoS measurement metrics needs to be designed. 


\section{PROPOSED SOLUTION}

In this section, against the research issues identified in Section 5, we propose the framework of a customized semantic service search engine (CSSSE) for the Digital Ecosystem environment. The overall architecture of the CSSSE is presented in Fig. 4.

As shown in Fig. 4, the CSSSE system can be mainly divided into two layers - system layer and web interface layer. The system layer is the core part of the system which implements most of the systemic functions. The web interface layer is the place where the system interacts with users. In the rest of the section, we will describe each component of the system in accordance with the proposed functions of the CSSSE.

\section{A. Service Knowledge Base}

Service knowledge base is designed to store the service domain knowledge, which can be divided into two parts service ontology and service description entity (SDE) metadata. A service ontology is the representation of the knowledge with regard to the taxonomy in a specific service domain, which concerns about the abstraction of the generic service concepts and their relationships between concepts from that domain, e.g., truck transport and road transport can be viewed as two concepts in the transport domain, and the former is the subclass of the latter. A SDE metadata is the representation of the knowledge with regard to an actual service entity, which concerns about the structuralized description to a service entity provided by a service provider, e.g., a truck transport service provided by a transport company can be represented by a SDE metadata. The indepth information concerning the service knowledge base can be referred from [14].

\section{B. Semantic Crawler}

Semantic Crawler is designed with the purpose of extracting SDE metadata from the web. The crawler is able to visit and download all the webpages under a given website. Once a webpage is obtained, the crawler can analyze the structure of web document, and then parses the web document into small pieces based on the predefined parsing rules. Next, meaningful information from the parsed web document is extracted and annotated by RDF/OWL in order to generate the eventual SDE metadata. There can be multiple semantic crawlers working in a multi-thread manner, which each crawler is able to communicate with others in order to avoid redundancy. The in-depth information about the semantic crawler can be referred from [15].

\section{Hybrid SDE Metadata and Service Concepts Association Module and Service Provider Web Interface}

The hybrid association module is designed in order to categorize the SDE metadata by means of the service ontology. This objective is realized by mutually assigning the URLs of metadata and service concepts to each other. The hybrid association module comprises an automatic and a manual association sub-module. The automatic association

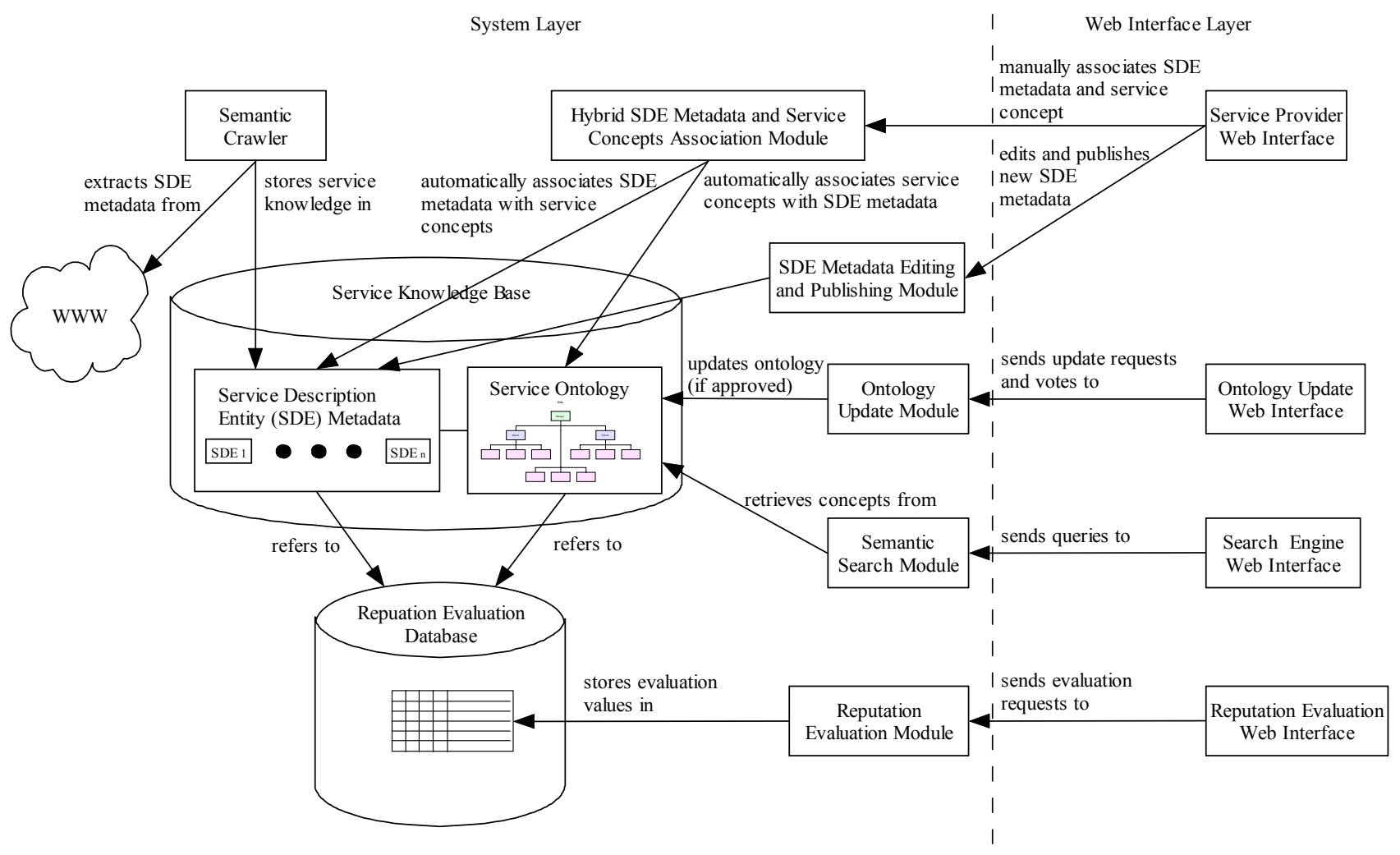

Figure 4. Overall architecture of the proposed CSSSE 
sub-module employs a concept-metadata similarity algorithm to compute the semantic similarity between concepts and metadata, then determines whether a concept and a metadata are semantically similar. The manual association sub-module makes use of the service provider web interface to allow a service provider to determine whether a belonged SDE metadata is semantically similar to a service concept. Obviously the former utilizes an objective matching method, and the latter utilizes a subjective one, where the latter is a useful complement of the former in order to achieve the most precise association result. The in-depth information regarding the hybrid association module can be referred from [16].

\section{SDE Metadata Editing and Publishing Module}

In addition to extracting metadata by the semantic crawler, service providers are also allowed to edit the belonged metadata and publish new metadata by means of the SDE metadata editing and publishing module. Similar to the crawler, the edited or published metadata is annotated by $\mathrm{RDF} / \mathrm{OWL}$ and stored in the service knowledge base.

\section{E. Ontology Update Module and Web Interface}

The ontology update module is designed to evolve the service ontology on community-based decisions. The evolution involves the changes of ontology concepts and changes of relationships between concepts. By cooperating with an ontology update web interface, a user can send an ontology update request to the module, and then a voting based on the request is built with a deadline. All registered users are allowed to vote for the request. The users are distinguished to two groups according to different user rights, which are domain experts and normal users. Once the deadline is approached, the total voting result will be computed by assigning different weights to the two groups of votes. If the total voting result surpasses a predefined threshold value, the ontology will be changed based upon the user's request; otherwise not.

\section{F. Semantic Search Module and Search Engine Web Interface}

The semantic search module is designed for service requesters to retrieve service providers who can provide a desired service. The module adopts a human-centered searching process in order to assist service requesters to denote the desired service concepts from service ontology. This is realized by cooperating with a search engine web interface. Once a service requester enters a query that normally consists of words connected by Boolean operations (and, or, not). The query words will be filtered and expanded by sending them to WordNet ${ }^{\circledR}$. The processed query words then are compared with each ontology concept in the knowledge base and the similarity values between the ontology concepts and the query are computed. If the similarity values are beyond a threshold, the belonged concepts can be recognized as matched to the query. Later on all the matched concepts are ranked and displayed to the service requester for selecting. The concept selecting is a recursive process: once a concept is selected, if it is an upper concept in the ontology, all its sub-concepts will be displayed for the further concept denotation; if it is a bottom concept, all its associated SDE metadata will be returned to the requester.

\section{G. Reputation Evaluation Module, Database and Web Interface}

There are two goals of designing the reputation evaluation module, database and web interface - to allow service requesters to measure the QoS regarding a SDE metadata after a service transaction and to rank the retrieved SDE metadata under a service concept based on QoS. To achieve the first goal, after a service requester completes a service transaction with a service provider, the service requester is authenticated to login the web interface to evaluate the QoS regarding the relevant SDE metadata. Here we adopt the CCCI (correlation, commitment, clarity and importance) metrics to measure the QoS of the service metadata. The service requester is allowed to give marks to each QoS criterion of the metadata according to the rules of CCCI metrics. The criteria are predefined and determined by the service concepts. In other words, difference concepts have different criteria. Once all of the criteria are marked, the trustworthiness value of the requester to the SDE metadata under the service concept is obtained by combing all of the marks. Then the reputation value of the SDE metadata under the service concept is acquired by averaging all of requesters' trustworthiness values towards it. Moreover, the reputation value towards each criterion is calculated by averaging all of the requesters' marks towards it. Eventually, the reputation value and QoS criterion reputation values of metadata are stored into the database and can be utilized as multi-linear ranking benchmarks of all the metadata under a service concept, which can be used to achieve the second goal. The in-depth information regarding the reputation evaluation module and CCCI metrics can be respectively referred from [17] and [18].

\section{CONCLUSIONS AND FUTURE WORKS}

In this paper, we study the research issues of semantic service retrieval and QoS measurement in the Digital Ecosystem environment. Digital Ecosystem emerges with the purpose of supporting the interactivities among species in the business ecosystem. Service transaction is one form of such interactivities. In the service interactions, species can play dual roles - service provider and service requester. Three issues are observed in the service interactions within the Digital Ecosystem environment, which are the mislocation of service providers, unstructured and semantic-less service information, and lack of unified QoS measurements. In order to further explain the research issues and to reveal our motivations, we use a case study that John searches for a sheep removal service from the existing commercial search engines. The case study results show that current commercial search engines meet difficulties in order to satisfy John's such requirement. This motivates us the desire that designs a semantic service search engine integrated with a unified QoS measurement for service evaluation and ranking within the Digital Ecosystem environment. Following that we make a 
survey on the existing literature in this field and find that none of these researches concerns about our research motivations and cannot satisfy the requirements of QoSbased service retrieval in the Digital Ecosystem environment. Therefore, we formally define the core research question how to design a multi-domain or specific domain service search engines in the Digital Ecosystem environment enhanced by semantic web technologies and QoS measurements, and its parallel potential research issues. In order to solve the research question and issues, we propose the conceptual framework of a CSSSE system, which integrates the functions of service knowledge base, semantic crawler, hybrid metadata and concept association, metadata editing and publishing, ontology update, semantic search and reputation evaluation.

Since the CSSSE project is a state-of-the-art work and its implementation is still undergoing, we are going to complete the project and implement the prototype in a specific service domain. The implementation can be divided into the following processes:

- We will collaborate with domain experts to create domain ontologies for the service knowledge base, and concept-based QoS measurement criteria.

- We will employ the semantic crawler(s) to collect SDE metadata for the service domain.

- We will invite service providers from the service domain to edit and publish services on the platform.

- We will call for service requesters' participation in the CSSSE platform.

- A series of experiments will be undertaken in order to evaluate the feasibility of those algorithms, mechanisms and rules employed in the CSSSE prototype.

\section{ACKNOWLEDGMENT}

We would like to express our gratitude for the assistance of all relevant DEBII staff, especially to our programmer Wei Liu who took responsibility for implementing the CSSSE prototype.

\section{REFERENCES}

[1] P. Dini, N. Rathbone, M. Vidal, P. Hernandez, P. Ferronato, G. Briscoe, and S. Hendryx, "The digital ecosystems research vision: 2010 and beyond," European Commission 2005.
[2] H. Dong, F. K. Hussain, and E. Chang, "Semantic service search, service evaluation and ranking in Service Oriented Environment," in IT Revolutions 08, Venice, Italy, 2008, pp. 111-117.

[3] J. F. Moore, The Death of Competition: Leadership and Strategy in the Age of Business Ecosystems. New York: Harper Business, 1996.

[4] E. Chang and M. West, "Digital Ecosystem - A next generation of the collaborative environment," in iiWAS2006, Yogyakarta, 2006.

[5] P. Dini, "Introduction - A Science Foundation for Digital Ecosystems " in Digital Business Ecosystems F. Nachira, A. Nicolai, P. Dini, M. L. Louarn, and L. R. Leon, Eds.: European Commission, 2007.

[6] D. R. Liu, M. Shen, and C. T. L, "Designing a composite e-service platform with recommendation function," Comput Stand Interfaces, vol. 25 , pp. 103-117, 2003.

[7] ITU-T Study Group, Teletraffic Engineering Handbook International Telecommunication Union, 2006.

[8] C. Aurrecoechea, A. T. Campbell, and L. Hauw, "A survey of QoS architectures," Multimedia Systems, vol. 6, pp. 138-151, 1998.

[9] J. Gekas, "Web service ranking in service networks," in the $3 r d$ European Semantic Web Conference (ESWC 2006), Budva, 2006.

[10] D. A. Menascé, "QoS issues in web services," IEEE Internet Computing, vol. 6, pp. 72-75, 2002.

[11] D. Roman and H. Lausen, "Web service modelling ontology(WSMO)," WSMO 2005.

[12] Y. Zhang, H. Huang, Y. Qu, and X. Zhao, "Semantic service discovery with QoS measurement in universal network," in LNAI: RSEISP 2007. vol. 4585, M. Kryszkiewicz, Ed. Heidelberg: SpringerVerlag Berlin, 2007.

[13] S. Pan, "A model for web services discovery with QoS," $A C M$ SIGecom Exchanges vol. 4, pp. 1-10, 2003.

[14] H. Dong, F. K. Hussain, and E. Chang, "Transport service ontology and its application in the field of semantic search," in 2008 IEEE International Conference on Service Operations and Logistics, and Informatics (SOLI 2008), Beijing, China, 2008, pp. 820-824.

[15] H. Dong, F. K. Hussain, and E. Chang, "A transport service ontologybased focused crawler," in International Conference on Semantics, Knowledge and Grid 2008 (SKG 2008), Beijing, China, 2008, pp. 4855.

[16] H. Dong, F. K. Hussain, and E. Chang, "A hybrid service metadata clustering methodology in the Digital Ecosystem environment," in The IEEE 23rd International Conference on Advanced Information Networking and Applications (AINA' 09) Bradford, UK: IEEE CS 2009, pp. 238-243.

[17] H. Dong, F. K. Hussain, and E. Chang, "Quality of service (QoS) based service retrieval engine," in The 6th International Conference on Advances in Mobile Computing and Multimedia (MoMM 2008), Linz, Austria, 2008, pp. 405-408.

[18] E. Chang, T. S. Dillon, and F. Hussain, Trust and Reputation for Service Oriented Environments-Technologies for Building Business Intelligence and Consumer Confidence: John Wiley \& Sons, 2005. 